\title{
Bronchoscopic management of tracheal lesions using the neodynium yttrium aluminium garnet laser
}

\author{
L TOTY, C PERSONNE, A COLCHEN, AND G VOURC'H
}

From the Departments of Thoracic Surgery and Anaesthesia, Hôpital Foch, Suresnes, France

\begin{abstract}
We report our first clinical trials with the NdYAG laser in the treatment of tracheal and bronchial tumours and stenoses. The beam is carried through a flexible fibre delivering a power of 50 to 90 watts. It can be introduced through the biopsy channel of a standard bronchoscope, or through a fibrescope. Anaesthesia must avoid inflammable gases. In most cases, general anaesthesia was used, and ventilation achieved with a mixture of $50 \%$ nitrogen and oxygen, using a modification of the Sanders injector. One hundred and sixty-four cases have been treated in 317 sessions (from one to five sessions per patient). They comprised: 72 cancers, 24 of which had just been diagnosed and had acute respiratory obstruction. In 16, one single session restored the patency of the airway. Forty-eight other cases were recurrent carcinomas after either surgery or radiotherapy, nine of which were caused by cancers of other origin invading the trachea $; 21$ benign or moderately malignant tumours; 44 iatrogenic stenoses, including 31 narrow ones. Of those 31 cases, 17 had an immediate satisfactory result, but eight recurred; 24 granulomas on bronchial suture lines. There were two deaths not directly related to surgery or anaesthesia. Bleeding was never more than moderate. The main difficulty lies in the critical respiratory condition of the patients, sometimes seen in acute asphyxia, referred to us as a last resort, especially those with carcinomas involving the trachea or main bronchi.
\end{abstract}

Thoracic surgeons and bronchoscopists often meet with difficult problems involving the trachea and bronchi, such as stenoses of neoplastic or inflammatory origin which are not easily managed by conventional techniques.

Emergency tracheostomy may be necessary. Tracheal resection is the technique of choice whenever practicable, or a Rethi procedure in difficult cases.

Bronchoscopic procedures are usually limited to coagulation or piecemeal removal which, combined, may restore the patency of the airway and buy time for further surgery. Those techniques involve risks, in particular of haemorrhage. They may worsen a stricture through scarring, and even lead to perforation, particularly in cases of repeated coagulation.

The laser beam offers many advantages including reduced bleeding and oedema, and quick cicatrisation. The carbon dioxide $\left(\mathrm{CO}_{2}\right)$ laser has been used with success in the management of laryngeal lesions. One case only has been reported of tracheal disobliteration using the $\mathrm{CO}_{2}$ laser. ${ }^{1}$ Unfortunately, the

Address for reprint requests: Dr L Toty, Department of Thoracic Surgery, Hôpital Foch, 92 Suresnes, France.
$\mathrm{CO}_{2}$ laser beam travels in a straight line and its application to tracheal and bronchial lesions is necessarily limited and not easily performed. It can aim only at the upper part of the trachea and cannot be expected to perform resections on the tracheal wall itself without major risks of perforation.

The neodynium yttrium garnet laser (NdYAG) overcomes this difficulty. It has been used in gastrointestinal tract lesions. ${ }^{2-4}$ The beam is carried through a flexible conductor and can be aimed in any direction under visual control using a rigid or a fibreoptic bronchoscope. This technique provides the best and safest conditions for endoscopic resections; under visual control, great accuracy is possible, and coagulation can be carried out as far down as required. One of the main advantages over conventional coagulation is that the tip of the fibre does not come into contact with the tumour, because that inevitably induces bleeding. The field is, therefore, completely clear. Furthermore, coagulation is limited to the surface of the lesion and does not spread in depth as does diathermy. We have applied it to the trachea and bronchi and are reporting our preliminary results on 164 patients. 


\section{Methods}

The apparatus used (Compagnie Industrielle des Lasers (CILAS), Route de Nozat, 91460 Marcoussi, France) provides an output of up to 90 watts in the infrared wavelength $(\lambda 1.06 \mathrm{~m} \mu)$. A combined pilot beam (helium and neon) produces a bright red spot which can be aimed towards the lesion.

These two beams are conveyed through the same flexible fibre, of $1 \mathrm{~mm}$ diameter. The core is made of silica, wrapped in a silicon sheath. Another coaxial teflon tube $(1.8 \mathrm{~mm}$ external diameter) allows the injection of compressed air $(31 / \mathrm{min})$ to cool the tip of the laser fibre, prevent condensation on the endoscope and disperse fumes.

Since the laser beam has a $10^{\circ}$ divergence, the diameter of the impact varies with the distance between the tip of the fibre and the target. In practice, that distance is kept between 5 and $10 \mathrm{~mm}$.

The laser fibre is inserted through the biopsy channel of a standard rigid bronchoscope, or can be used with a T3 Olympus flexible fibrescope fitted with a $2.7 \mathrm{~mm}$ channel. In either case, the fibre can be bent in any direction and aimed towards the lesion.

Power, time, and distance from the lesion can be adjusted according to the case considered: coagulation-30 watts, for 0.7 to $1 \mathrm{~s}$, over a surface of about $3 \mathrm{~mm}$, at a distance of $10 \mathrm{~mm}$; excision -40 to 90 watts, for $0.3 \mathrm{~s}$ in bursts of one every two seconds, at a distance of about $5 \mathrm{~mm}$.

Each burst triggers a sound signal, and a counter records the number of bursts. A foot switch worked by the operator controls first the blast of compressed air then the laser itself.

Since the laser beam can induce severe burns, particularly of the eyes, ${ }^{5}$ special goggles (CILAS) must be worn by all the attending personnel. They provide a reduction of power of $10^{6}$ for $\lambda 1.06 \mathrm{~m} \mu$.

The energy delivered by the NdYAG laser is better absorbed by dark surfaces (melanin, haemoglobin). It may, therefore, be necessary to apply a dye, such as methylene blue, to any white structure before starting the laser.

\section{ANAESTHESIA}

This application of the NdYAG is painless. But in order to provide the bronchoscopist with optimal conditions, and to ensure accurate aiming, coughing and struggling must be avoided. Topical analgesia, supplemented by sedation induced by various drugs, such as opiates, is adequate in certain cases. But general anaesthesia and mechanical ventilation are often necessary, particularly in patients in respiratory distress.

In the adult, and if a fibrescope is available, the procedure may be carried out under tracheal intubation, using a large, cuffed tube. The fibrescope is inserted through a rubber diaphragm within the tracheal tube and ventilation achieved either by manual or mechanical control. ${ }^{6}{ }^{7}$ In all other casesthat is with children or when a rigid bronchoscope is used-lung inflation using the Sanders injector was found satisfactory, but the apparatus had to be modified to meet the situation. The standard injector fitted at the proximal (machine) end of the bronchoscope is inadequate, because the bulk of the laser fibre and the leaks through the side vents of the bronchoscope, especially if the lesions are situated just below the cords, resulted in inadequate ventilation. Therefore a 16 gauge brass tube was welded on the side of the bronchoscope, opening at the tip, and within the lumen to avoid damage to the mucosa by the jet of compressed gases. This device has been used in nearly all our cases, and found to be entirely satisfactory.

Because of the intense heat generated by the laser beam, inflammable gases must not be used. Cases of fire have been reported using the $\mathrm{CO}_{2}$ laser, ${ }^{8}{ }^{9}$ and adequate protection must be achieved to prevent combustion of rubber or plastic material. In practice, under general anaesthesia, the following technique is used: after standard premedication, anaesthesia is induced using a sleep dose of thiopentone and/or an intravenous infusion of short acting agents such as alfathesin, methohexital, or propanidid to ensure unconsciousness throughout the procedure, and muscle relaxation achieved by a suxamethonium infusion. Ventilation is carried out with an equal mixture of nitrogen and oxygen, which was found to be safe. It would be very tempting to use high frequency positive pressure ventilation ${ }^{10}$ which would avoid the spread of blood into the distal bronchial tree by the jet of gases, and it is our intention to try it. There may be a danger, however, of accidental airway obstruction passing unnoticed, since there is no chest expansion for the anaesthetist to observe.

\section{Preliminary trials}

Before the clinical application, 27 experiments with the NdYAG laser were made on dogs. They included excision of granulomas previously induced by irritation of the tracheal mucosa, or developing on sutures after bronchial resection and anastomosis. It was found that cicatrisation was achieved in eight to 10 days, and that induced haemorrhage was easily controlled.

Pathological examination of the laser-induced lesion ( 50 to 90 watts for $0.3 \mathrm{~s}$ ) on healthy mucosa showed some degree of inflammatory reaction, but the underlying tissues were hardly affected and smooth muscle was spared. No perforation or secondary necrosis were observed. 
Clinical results (table)

During the past 18 months (November 1978-May 1980), 164 patients have been treated. This corresponds to 317 operative sessions, since most patients had to be submitted to the procedure more than once. Ninety-five patients had one session, the rest between two and five. It is too early yet, from such a short series, to draw definite conclusions but this new technique appears very promising.

\section{Table Conditions treated}

\begin{tabular}{lllr}
\hline Category & Number & Type & Number \\
\hline Cancers & 72 & Primary untreated tracheal & 24 \\
& & cancers & 48 \\
Benign & 11 & Recurrent cancers & 1 \\
tumours & & Histiocytoxanthofibroma & 1 \\
& & Solitary papillomas & 3 \\
& & Leiomyoma & 1 \\
& & Diffuse papillomas & 3 \\
Moderately & 10 & Diffuse amylosis & 2 \\
malignant & & Carcinoids & 7 \\
tumours & & Cylindroma & 3 \\
Iatrogenic & 47 & & 47 \\
stenosis & & & 24 \\
Granulomas & 24 & & 164 \\
\hline
\end{tabular}

TRACHEAL OR BRONCHIAL CARCINOMA (72 CASES)

Two groups of cases have been treated.

\section{Tracheal carcinomas recently detected (24 cases)}

These tumours had been diagnosed after acute respiratory distress previously attributed to other causes. In nearly all cases emergency intubation or tracheostomy had to be performed before radiotherapy. In 16 cases, a single session immediately restored adequate respiratory function. Particularly striking was the case of a patient who on his way to hospital had sustained two episodes of anoxic cardiac arrest, and who was discharged the following day in excellent respiratory condition. The other cases required a second session. In five, intubation was considered necessary for 24 hours. Tracheostomy was considered necessary in only one case.

\section{Recurrent carcinomas (48 cases)}

Patients from this group were referred to us because of intractable bronchial obstruction. Some had been operated on previously (18 cases, including six pneumonectomies); the others had been considered inoperable. Nearly all of them had been irradiated, and some were having chemotherapy. For this group of patients, laser surgery is indicated only if the respiratory obstruction is the only, or the main problem. The removal of a tumour involving the trachea or the carina, on a bronchial suture line, or in one main bronchus, may relieve the patient and provide him with some months of comfort. The procedure is obviously purely palliative and has to be repeated whenever required. Nine patients from this group, first seen in acute respiratory distress, are now alive a year later, after two to five sessions. Eight are still alive or were kept alive for six months. For all of them, laser treatment was the only solution.

Among this group there were nine cases of carcinomas invading the trachea from the oesophagus (seven cases) or the thyroid (two cases).

\section{BENIGN TUMOURS (11 CASES)}

True benign tumours of the trachea or main bronchi are very rare. We have treated by laser six solitary tumours (three papillomas, one amyloid, one histiocytoxanthofibroma, one leiomyoma). None have recurred so far, but the follow-up period is still short. In this group, laser treatment may provide complete relief, avoiding major surgery.

Five cases of benign diffuse tumour (three papillomatoses, two amyloids) benefited from partial disobliteration. Laser treatment can only ablate the main part of the growth.

MODERATELY MALIGNANT TUMOURS ( 10 CASES, INCLUDING SEVEN CARCINOIDS AND THREE CYLINDROMAS)

These tumours are obvious indications for surgery, but some are not operable, or the risk of operation may be excessive considering the slow rate of growth.

Four of our patients were high risk cases (respiratory, cardiac, or renal failure in elderly patients). Three had intractable recurrences after operation (tracheal recurrence after pneumonectomy for cylindroma, carinal recurrence of cylindroma after lobectomy for carcinoid). Among cases operated on for more than six months, there has been only one recurrence.

In all these cases, recurrence is expected, and laser treatment can only remove the stem of the tumour. Three of the carcinoid tumours were amenable to surgical treatment, but the patients refused operation. One has not yet recurred; the other two have now been operated on.

\section{IATROGENIC STENOSIS (47 CASES)}

These vary considerably in their site (from the subglottic region to the lower third of the trachea), the date of their appearance (from a few weeks to some years), their extension (simple diaphragm or a long cylinder), and their size. They often affect young, otherwise healthy patients, hence the importance of relieving them. 
It is obvious that the laser beam cannot attack more than the fibrous tissue implanted on the tracheal wall itself. As seen through the bronchoscope, one gets the impression of a funnel-like lesion sprouting from an endotracheal stenosis.

Two types of stenoses can be recognised-the fibrous diaphragm, sparing the tracheal wall, and one involving the trachea itself, with narrowing, collapse, and sometimes fracture of the cartilage rings. The first type is rare, and can be radically cured by laser treatment. The second is far more common and cannot be treated by endoscopy. In most cases, however, the two are combined, the stenosis evolving both from the mucosa and submucosa.

The results achieved by laser treatment depend upon which form is predominant. We shall discard 16 cases of minor stenosis (less than $50 \%$ of the lumen) which might not have been treated, and consider only 31 cases of narrow strictures ( 3 to $4 \mathrm{~mm}$ ) for which treatment was obligatory. In 17 cases, nearly complete disobliteration was achieved ( $75 \%$ of the lumen), while in the worst cases (recurrent stenosis after one or many surgical procedures such as resectionanastomosis), the lumen was doubled in area.

During a follow-up period of six to 18 months, eight of the 17 cases first considered to be satisfactory recurred and had to be operated on. Nine only may, therefore, be considered as cured.

\section{GRANULOMAS (24 CASES)}

The laser is the ideal technique for the cure of granulomas developing on a tracheal or bronchial suture line, or after prolonged tracheal intubation. They must not be neglected since they may lead to a fibrous stenosis. Four of these granulomas, the size of a hazelnut, caused considerable obstruction of the trachea or main bronchus.

\section{Technical problems and complications}

The technical problems of endoscopic laser surgery are now easily mastered. Haemorrhage, particularly when dealing with malignant growth, can be disturbing, through blurring the optic, but has never been severe (10 to $20 \mathrm{ml}$ at the most).

We have not seen the development of a bronchooesophageal fistula. Two cases of pneumothorax, due to jet inflation, were easily managed.

Two patients died in the immediate postoperative period. One (iatrogenic tracheal stenosis) died about 10 hours after the procedure. Although she appeared to have been relieved, she went into respiratory distress and died of asphyxia in the recovery ward. Neither the laser nor anaesthesia, were involved, but resuscitation seems to have been inadequate. The other case was that of an 89-year-old woman with tracheal cancer and metastasis, who died three days after the procedure, from lung infection. No resuscitation was undertaken.

This was the total mortality and morbidity in the 164 patients treated. The patients were mostly high risk cases submitted to 317 anaesthesia procedures, and often with critical respiratory obstruction.

\section{Discussion}

In our experience, NdYAG laser endoscopic surgery appears to be a safe procedure, and more accurate and less dangerous than conventional techniques $\dot{\omega}$ such as coagulation by diathermy. It is mainly an $\vec{v}$ adjunct to surgery, in inoperable cases, particularly those of recurrent cancers, but it cannot replace surgery, except in some cases of iatrogenic stenosis and benign tumours. Its main and most interesting indication lies in the treatment of asphyxiating, and intractable forms of cancers, previously operated on and irradiated. In such cases it provides relief and prolongs the patient's life with a minimum of risk at the cost of a short stay in hospital.

\section{References}

1 La Foret EG, Berger RL, Vaughan CW. Carcinoma obstructing the trachea. Treatment by laser resection. N Engl J Med 1976; 294:941.

2 Fruhmorgen P, Bordem F, Reidenbach HD, Kaduk B, Demling L. Endoscopic laser coagulation of bleeding gastrointestinal lesions with report of the first therapeutic application in man. Gastrointest Endosc 1976; 23:73-5.

3 Kief haber P, Nath G, Moritz K. Endoscopic control @ of massive gastrointestinal hemorrhage by irradiation with high power neodynium YAG laser. Prog 3 Surg 1977; 15:140-55.

4 Brunetaud J, Enger A, Maffioli C, Seys A, Potron G, Berjet M. L'hémostase per-endoscopique par le laser des hémorragies digestives d'origine haute: premiers résultats. Nouv Presse Med 1978; 7:1486.

5 Leibowitz HM, Peacock GR. Corneal injury produced by carbon dioxide laser radiation. Arch Ophthalmol 1969; 81:713-21.

6 Tahir AH. General anesthesia for bronchofiberos- N copy. Anesthesiol 1972; 37:564.

7 MacNaughton FR. Anaesthesia for fiberoptic $\mathrm{C}$ bronchoscopy. Br J Anaesth 1975; 47:1219-23.

8 Snow JC, Norton ML, Saluja TS, Estalina J A.F. Fire

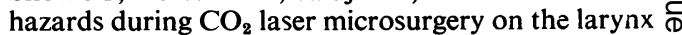
and trachea. Anesthes Analges Curr Res 1976; $\stackrel{\circ}{+}$ 55:146-7.

9 Vourc'h G, Tannieres ML. Ignition of a tracheal tube during laryngeal laser surgery. Anaesthesia 1979; $\mathbb{\mathbb { D }}$ 34:685.

10 Sjostrand U. Experimental and clinical evaluation of $\stackrel{\mathbb{Q}}{\Omega}$ high frequency positive-pressure ventilation (HFPPV) Acta Anaesthesiol Scand 1977; supplement 64. 